\title{
The Implicit Relational Assessment Procedure (IRAP) as a measure of obsessive beliefs in relation to disgust
}

\author{
Emma Nicholson*, Angela McCourt, Dermot Barnes-Holmes \\ National University of Ireland, Department of Psychology, John Hume Building NUI Maynooth, Maynooth Co. Kildare, Ireland
}

\section{A R T I C L E I N F O}

\section{Article history:}

Received 12 October 2012

Received in revised form

19 February 2013

Accepted 27 February 2013

\section{Keywords:}

Disgust

Implicit

Obsessive belief domains

Obsessive-Compulsive Disorder

\begin{abstract}
A B S T R A C T
The Implicit Relational Assessment Procedure (IRAP) was utilized as a means of interpreting disgust in terms of the six domains of Obsessive-Compulsive Disorder as defined by the Obsessive Compulsive Cognitions Working Group; excessive responsibility, overestimation of threat, perfectionism, intolerance for uncertainty, over importance of thoughts and need to control thoughts. A non-clinical sample ( $N=44$ undergraduate students) completed an IRAP designed to assess appraisals of disgust-inducing pictorial stimuli based on the six belief domains at the implicit level. A series of self-report measures including the Obsessive-Beliefs Questionnaire, the Padua Inventory and the STAI were also implemented. Results indicated that a greater bias toward appraising disgusting stimuli as being negative was related to excessive responsibility and overestimation of threat along with perfectionism and intolerance for uncertainty. Critically, these effects were found to be independent of anxiety supporting the influence of disgust responding in the etiology of OC tendencies.

( 2013 Association for Contextual Behavioral Science. Published by Elsevier Inc. All rights reserved.
\end{abstract}

\section{Introduction}

The role of disgust in the etiology of psychopathologies such as Obsessive-Compulsive Disorder (OCD) has been the focus of much research in the last decade. Disgust is a universally experienced negative emotion which has implications for the cognitive and behavioral domains of OCD. Many experiences may elicit disgust, for example, body-envelope violations, experiences involving animals or body waste or particular socio-moral scenarios (Haidt, McCauley \& Rozin, 1994). Measures (both implicit and explicit) have been developed to assess individual differences in general disgust and its two constituents, disgust propensity (i.e., an individual's tendency to experience disgust) and sensitivity (i.e., how negatively the individual appraises their experience of disgust) (Nicholson \& Barnes-Holmes, 2012a; van Overveld, De Jong, Peters, Cavanagh \& Davey, 2006). Numerous studies have utilized such measures in an attempt to delineate the influence of disgust, as an individual emotion, in the etiology of OCD.

Disgust has been related to general OCD symptoms and washing concerns in OCD, using self-report measures and this effect was independent of anxiety (Olatunji et al., 2007). Moretz \& McKay (2008) demonstrated that disgust influences general OCD symptoms and beliefs, including washing concerns and contamination fears, without any influence of anxiety. Thus, it appears

\footnotetext{
* Corresponding author. Tel.: +3531708 6086; fax: +35317084767.

E-mail address: Emma.Nicholson@nuim.ie (E. Nicholson).
}

from the literature that disgust does not merely influence the symptomatology of OCD through anxiety, rather it is a distinct emotion worthy of individual empirical investigation. While the evidence is promising, the specific cognitive mechanisms through which disgust influences OCD remain largely unclear. Cognitive approaches to obsessions posit that it is the misinterpretation of intrusive thoughts, feelings and images as being highly important which drives problematic behavior such as avoidance, reassurance seeking and excessive washing (Rachman, 1997, 1998; Salkovskis, 1985; Salkovskis, Shafran, Rachman \& Freeston, 1999). Thus, it may be that overtly negative interpretations of the initial feeling of disgust result in behaviors specific to OCD such as excessive washing and checking (Teachman, 2006).

The Obsessive Compulsive Cognitions Working Group (1997) have highlighted the relevance of cognitive content and processes in the etiology and maintenance of OCD. Critically, they have emphasized the importance of the interpretations (e.g., beliefs and appraisals) that follow intrusive thoughts such as "For me, having bad urges is as bad as actually carrying them out" or "Even if harm is unlikely, I should try to prevent it at any cost" Obsessive Compulsive Cognitions Working Group (2005). These background beliefs provide a context in which the intrusive thought is more likely to cause distress which result in the individual engaging in compulsive and problematic behaviors as a means of reducing this distress (Rachman, 1998). The OCCWG has identified six cognitive belief domains of OCD (1) excessive responsibility; (2) overestimation of threat; (3) perfectionism; (4) intolerance of uncertainty; (5) over-importance of thoughts and (6) need to 
control thoughts Obsessive Compulsive Cognitions Working Group (2001). These six domains have been narrowed down to three factors which can be measured by the Obsessive Belief Questionnaire, (1) responsibility/overestimation of threat; (2) perfectionism/intolerance of uncertainty; (3) over-importance/need to control thoughts (OBQ-44; Obsessive Compulsive Cognitions Working Group, 2005). These beliefs lead individuals to appraise otherwise harmless thoughts, feelings and images as being harmful and dangerous (Wu \& Carter, 2008).

Teachman (2006) argued that these cognitive domains provide a useful platform on which to establish the interpretation processes at work in disgust. Evidence from the literature suggests there are inconsistencies regarding the exact nature of the relationship between disgust responding, contamination fear and cognitive belief domains. For instance, Moretz \& McKay (2008) found that disgust propensity as measured by the Disgust Scale (van Overveld, De Jong, Peters \& Schouten, 2011) was related to obsessive beliefs. David, Olatunji, Armstrong, Ciesielski, Bondy \& Broman-Fulks (2009) found that disgust sensitivity failed to remain a significant predictor of OCD symptoms when controlling for obsessive beliefs (as measured by the OBQ). Evidence from Cisler, Brady, Olatunji \& Lohr (2010) suggests that cognitive beliefs may influence the role played by disgust in contamination fear, but this evidence is based on disgust propensity, which is the initial intrusive feeling of disgust. To the best of our knowledge, no previous study has attempted to delineate the relationship between disgust and obsessive beliefs by specifically measuring obsessive beliefs (as measured by the OBQ) in response to disgust-eliciting stimuli.

The IRAP is a computer-based procedure which requires participants to respond accurately and rapidly to sets of stimuli in a manner that is consistent or inconsistent with their previous learning history. It was derived from a modern behavior-analytic account of human language and cognition called Relational Frame Theory (RFT; see Hayes, Barnes-Holmes \& Roche, 2001). The basic assumption of RFT is that the fundamental components of human language and cognition are relational, and thus the IRAP focuses on assessing relations between stimuli (e.g. Hughes, Barnes-Holmes \& De Houwer, 2011). The unit of measurement is response latency from the presentation of the stimuli to the emission of a correct response. The fundamental hypothesis is that responding should be quicker across blocks of trials that are consistent rather than inconsistent with a previously established bias. The IRAP provides an advantage over other so-called implicit measures as it can assess propositional relations between concepts rather than mere associations (see Hughes et al., 2011 for a detailed treatment of this issue).

The fundamental IRAP effect which posits that responding should be quicker on bias consistent relative to bias-inconsistent trials has been explained in terms of the Relational Elaboration and Coherence model (REC; Barnes-Holmes, Barnes-Holmes, Stewart, \& Boles, 2010). The REC model assumes that brief and immediate relational responses (BIRRs) will occur on most trials of the IRAP before a participant presses a response key. These responses will be based on historical and existing contextual variables, with the most likely response being emitted first (Barnes-Holmes et al., 2010). This effect has been demonstrated in numerous studies pertaining to, for example, self-esteem (Vahey, Barnes-Holmes, Barnes-Holmes \& Stewart, 2009), spider fear (Nicholson \& Barnes-Holmes, 2012b), sexual attraction to children among sexual offenders (Dawson, Barnes-Holmes, Gresswell, Hart \& Gore, 2009) and cocaine dependence (Carpenter, Martinez, Vadhan, Barnes-Holmes \& Nunes, 2012), to name a few.

Implicit measures offer many advantages over traditional selfreport measures in the study of anxiety due to the seemingly uncontrollable and often conflicting nature of anxious phenomena, such as obsessions. In effect, the uncontrollable nature of anxiety seems to overlap with the types of psychological phenomena that implicit measures were designed to target (see Wiers, Teachman \& De Houwer, 2007). Critically, research suggests that participants have limited control over their responses on the IRAP (e.g., Dawson, et al., 2009; McKenna, Barnes-Holmes, BarnesHolmes \& Stewart, 2007). Further, recent evidence suggests that implicit measures may be able to provide insight into psychological constructs, the exact nature of which was previously unclear. A study on disgust using the IRAP demonstrated the utility of the IRAP at providing construct validity for an emotion (i.e., disgust) that had been previously ill-defined (Nicholson \& Barnes-Holmes, 2012a). Thus, implicit measures appear to offer promising methodologies for measuring the cognitive mechanisms underlying anxiety disorders such as OCD.

Increasing evidence from research using the IRAP suggests that it is the appraisal that follows the initial disgust-related thought that is indicative of avoidance behavior rather than the initial thought itself (e.g., Nicholson \& Barnes-Holmes, 2012a). This research utilized two IRAPs, one for disgust propensity and another for disgust sensitivity. Results demonstrated that each construct had a relationship with OC tendencies; however, these relations were different at the behavioral and symptomatic levels. It was found that disgust sensitivity predicted avoidance behavior independently of anxiety and was related to OC tendencies, specifically washing concerns. On the other hand, disgust propensity predicted OC tendencies and obsessing symptoms, independently of anxiety. This evidence suggests that not only can disgust interpretations be measured by the IRAP but they can be used to predict avoidance behavior and OC symptoms. However, this study merely examined disgust as being fearful, worrisome and intolerable with no reference to the cognitive belief domains specific to OCD such as excessive responsibility, perfectionism, overestimation of threat, intolerance of uncertainty and the need to control thoughts. As such the IRAP may offer a way of assessing the relevance of the obsessive beliefs domains in disgust responding.

The finding that appraisal of an initial feeling or intrusive thought appears to be a fundamental aspect of OCD provides support for both the seminal work of Salkovskis' (1985) on obsessions and more recent conceptual analyses arising from Acceptance and Commitment Therapy (ACT). Specifically, Salkovskis argued that intrusive thoughts, in the context of OCD carry little no valence until they are positively, negatively or neutrally appraised. Similarly, the ACT model suggests that treatment should target responses to cognitive experiences (such as intrusive thoughts) rather than specific content or emotions (Twohig, 2009). According to both views, therefore, it is not the initial reaction to OCD-relevant stimuli, but the reaction to the reaction that is key in defining and perhaps treating OCD itself. Thus research which builds on that of Nicholson \& Barnes-Holmes, 2012b could be seen as being directly relevant to both "traditional" CBT work in this area as well as "third-wave" behavior therapies.

The current study aimed to assess obsessive beliefs, as measured by the OBQ-44, in relation to disgust at the implicit level. The IRAP was used as a means to measure appraisals pertaining to obsessive beliefs in response to both disgust-eliciting and generically pleasant pictorial stimuli as well as positive descriptive words in response to the same stimuli. Questionnaires assessing obsessive beliefs, general OC tendencies and anxiety were also implemented as a comparison to the IRAP. As this was the first study to assess disgust in this manner, we refrained from making specific predictions. However, it was assumed that those who score highly on the explicit measures, specifically the OBQ, would produce greater implicit negative appraisals of the disgusting stimuli. 


\section{Methods}

\subsection{Participants}

There were no selection criteria in order to take part in this study with regards levels of disgust or OC tendencies. There is increasing support for the idea that OCD symptoms originate in normal human processing. Therefore, the use of non-patient samples that score high on self-report measures of OCD may be relevant to understanding the development of OCD (see Burns, Formea, Koertege \& Sternberger, 1995). Participants $(N=44$; 13 men and 31 women) were selected from the student population of NUI Maynooth. The mean age of the participants was 26.7 with a range of 18-46 years. Each participant provided written informed consent prior to taking part in the study and completed the experiment individually in the Department of Psychology at NUI Maynooth.

\section{Materials}

\subsection{Obsessive Belief Questionnaire-44 (OBQ-44; OCCWG, 2005)}

The Obsessive Belief Questionnaire is a 44 item self-report scale designed to measure individual differences in obsessive beliefs across three cognitive domains of OCD. It consists of three factors including (1) responsibility/threat (e.g. "If I do not take extra precautions, I am more likely than others to have or cause a serious disaster."), (2) perfectionism/uncertainty (e.g. "In order to be a worthwhile person, I must be perfect at everything I do."), (3) importance/control of thoughts (e.g. "If I have aggressive thoughts or impulses about loved ones, this means I may secretly want to hurt them."). Each item is rated on a 7-point (1-7) Likert scale of agreement with belief statements. Internal consistency achieved in this sample was approaching excellent $(\alpha=.785)$.

\subsection{Obsessive-Compulsive Inventory-Revised (OCI-R; Foa et al., 2002)}

The OCI-R is an 18 item self-report measure of symptoms of obsessive-compulsive disorder and was used to measure OC tendencies. It has successfully differentiated between individuals with and without OCD. For a non-anxious sample, it has demonstrated good-excellent internal consistency $(\geq .72)$, and testretest reliability $(.57-.87)$.

\subsection{Padua inventory-Washington State University Revision (PI-WSUR; Burns, Formea, Koertege E Sternberger, 1995)}

The Padua Inventory-Washington State University Revision is a 39 item self-report scale designed to measure obsessive and compulsive symptoms. It is also designed to reduce overlap with worry. Each item is rated on a 5-point (0-4) Likert scale assessing the degree of disturbance caused by thought or behavior. It consists of five subscales including (1) contamination obsessions and washing compulsions, (2) dressing/grooming compulsions, (3) checking compulsions, (4) obsessional thoughts of harm to self/others and (5) obsessional impulses of harm to self/others. This scale has adequate internal consistency and test-retest reliability (Burns, Koertege, Formea, \& Sternberger, 1996). Internal consistency achieved in this sample was approaching excellent $(\alpha=.711)$.

\subsection{State-Trait Anxiety Inventory-Form Y-1 (STAI; Spielberger, Gorsuch, Lushene, Vagg E' Jacobs, 1983)}

The State-Trait Anxiety Inventory-Form Y-1 is a 20 item selfreport subscale of the STAI designed to measure state anxiety, i.e. how an individual is feeling right now. Each item is rated on a 5-point (0-4) Likert scale of the level of present anxiety. The STAI has been found to have good reliability and validity (Spielberger et al., 1983).

\subsection{Implicit Relational Assessment Procedure (IRAP)}

The IRAP is a computer based measure which presents sets of stimuli in the form of trials within a series of blocks. On each trial of the IRAP, a label stimulus was presented at the top of the screen. There were a total of twelve label stimuli which were digital images; six were color photographs of things which would evoke disgust and the other six were color photographs of generically pleasant images. All but one of the label stimuli were taken from the International Affective Picture System ${ }^{1}$ (IAPS; Lang, Bradley \& Cuthbert, 1996). There were a total of twelve target stimuli, six of which referred to a negative appraisal based on the six cognitive belief domains of OCD (e.g. "My Responsibility", "I Must Control") and six of which referred to a positive response (e.g. "Soothing", "Harmless"). On each trial of the IRAP, one of the target stimuli was presented in the middle of the computer screen. Two response options ("True" and "False") were presented on each trial which appeared at the bottom left- and right-hand corners of the computer screen, alternating at random between trials (see Fig. 1).

The IRAP was presented in blocks of 24 trials which encompassed four different trial-types; Disgust-Negative Appraisal, Disgust-Positive Appraisal, Pleasant-Negative Appraisal, PleasantPositive Appraisal. Up to 6 practice blocks were implemented to ensure an accuracy rate of $80 \%$ and a response latency of less than or equal to $2000 \mathrm{~ms}$. The participants were required to meet these criteria across a pair of practice blocks, within six or less blocks, before proceeding to the fixed set of six test blocks. For each block of 24 trials, all of the label and target stimuli were presented in various combinations, with the program ensuring that each of the four trial-types was presented six times in a quasi-random order. The program also ensured that the same trial-type was not repeated across successive trials Table 1 .

The instructions for the IRAP were presented on paper and were read through with the experimenter to ensure that participants understood the nature of the experiment and what was being asked of them. The experimenter stressed the importance of speed and accuracy in the IRAP. Each participant was aware that, at times, they would be required to respond in a manner that may be consistent with their own beliefs and sometimes in a manner that may be inconsistent with their beliefs. The experimenter explicitly classified the pictorial stimuli as "disgusting" or "nondisgusting" thus providing a context to reduce the likelihood that participants would respond to the stimuli as being merely negative or positive. Furthermore, the response options "True" and "False" would likely serve to establish the relations between the pictures and statements as either relationally coherent or incoherent. For example, responding "True" to a disgusting picture and the statement "My Responsibility" asks a participant to respond to the relationship between the two items as relationally coherent; responding "False" in this case asks a participant to indicate that the relationship is incoherent (see BarnesHolmes, Hayes, Dymond \& O'Hora, 2001, for a more detailed treatment of "Truth" versus "Falsity"). Or in other words, the response options asked participants to evaluate or "appraise" the relational network in which the pictures and statements

${ }^{1}$ The picture numbers from the IAPS were as follows: $1201,9405,1440,1463$, $1710,5731,5760,1114,5780,3250,9300$. The twelfth picture was a photo of a dirty kitchen. 


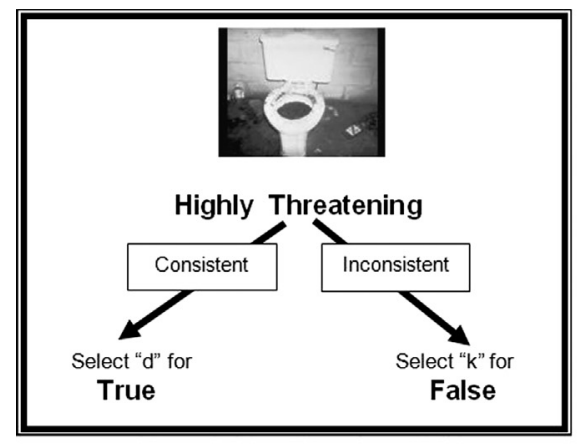

Disgust Negative-Appraisal

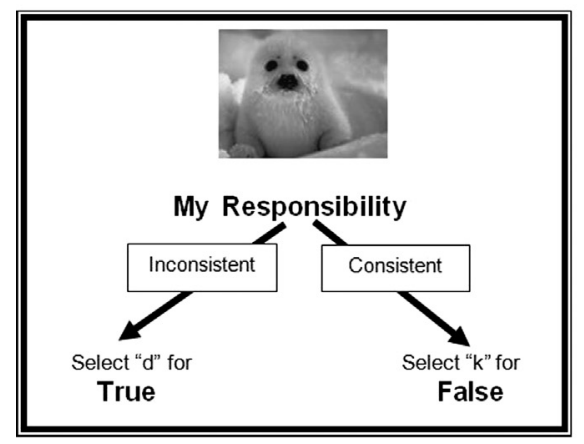

Pleasant Negative Appraisal

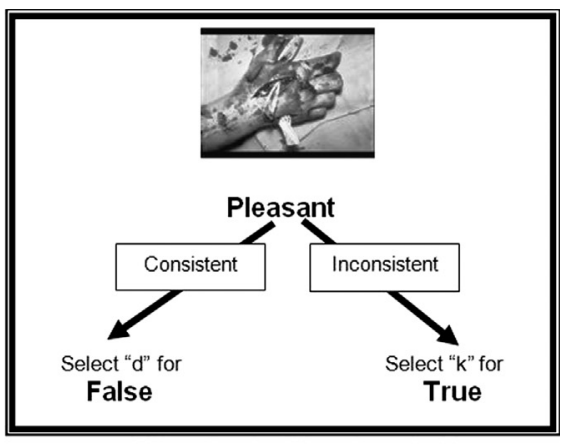

Disgust -Positive Appraisal

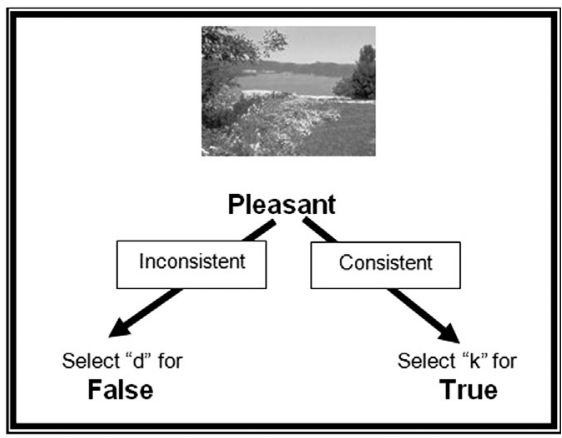

Pleasant-Positive Appraisal

Fig. 1. Examples of the four trial-types from the IRAP. Note. The boxed words ("Consistent" and "Inconsistent") and the arrows did not appear on screen for participants.

Table 1

Target stimuli presented in the IRAP.

\begin{tabular}{ll}
\hline Target stimuli & \\
\hline $\begin{array}{l}\text { Disgust-related } \\
\text { stimuli }\end{array}$ & $\begin{array}{l}\text { Pleasant-related } \\
\text { stimuli }\end{array}$ \\
\hline My responsibility & Acceptable \\
Highly threatening & Soothing \\
I must control & Pleasant \\
Unacceptable & Harmless \\
Not perfect & Perfect \\
Distressing & Not distressing \\
\hline
\end{tabular}

participated as either true or false. Participants were told that there were two rules that they would be required to respond in accordance with throughout the IRAP task (i.e., one rule for the consistent blocks and another for the inconsistent blocks). Thus, the participants were aware of the pattern of responding that would be required of them throughout the task before they began.

\subsection{General procedure}

Following informed consent, half the participants were selected randomly to complete the self-report questionnaires first, followed by the IRAP, with the remaining participants completing the measurements in the opposite order. The order in which the questionnaires were administered was randomized.

\section{Results}

\subsection{Scoring the IRAP}

The response latency, defined as the time in milliseconds ( $\mathrm{ms}$ ) from the onset of a trial to the first response, provides the primary datum from the IRAP. The IRAP effect is the difference between the consistent and inconsistent mean response latencies recorded for a specific trial-type. To insure that IRAP effects were derived from performances that involved the targeted patterns of stimulus control, response accuracy was assessed first. If accuracy fell below $70 \%$ on a given test block, or pair of test blocks, then the IRAP effects for that participant were calculated using the remaining two pairs of test blocks. The data for two participants were analyzed in this manner. If accuracy fell below 70\% across multiple pairs of tests blocks then the data for that participant were discarded - the data for one participant were removed on this basis.

The latency data were transformed into D-IRAP scores using the adapted version of the Greenwald, Nosek \& Banaji (2003) $D$-algorithm (see Nicholson \& Barnes-Holmes, 2012b, for a full description of this procedure). The $D$-algorithm is used to minimize the impact of extraneous factors such as age, motor skills, and/or cognitive ability (Nosek, Greenwald \& Banaji, 2007).

Given the foregoing transformation, a larger $D$-IRAP score indicates a greater difference in response latencies between consistent and inconsistent trials. Positive scores indicate responding in accordance with expected response biases (e.g., responding "True" more quickly than "False" when a disgusting picture appeared with a negative appraisal) and negative scores indicate responding in a manner that was inconsistent with expected responses biases (e.g., responding "False" more quickly than "True" when a disgusting picture appeared with a negative appraisal). Scores that approach zero indicate no difference between consistent and inconsistent test blocks.

\subsection{Implicit measure analysis}

Scores from each trial-type in the IRAP were in the expected direction and were significant from zero, Disgust-Negative Appraisal $(M=.284, S D=.332 ; t(26)=4.43, p<.0001)$; DisgustPositive Appraisal $(M=.343, S D=.333 ; t(26)=5.35, p<.0001)$; 
Table 2

Pearson correlation matrix of Disgust-Negative Appraisal trial-type, OBQ, OCI-R, PI and STAI.

\begin{tabular}{|c|c|c|c|c|c|c|c|c|c|c|c|c|}
\hline Variable & Disgust-Neg & OBQ & $R / T$ & $\mathbf{P} / \mathbf{C}$ & $\mathbf{I} / \mathbf{C T}$ & OCI-R & PI & Con & $D / G$ & $\mathrm{C} / \mathrm{C}$ & $\mathbf{0} / \mathbf{T}$ & $\mathbf{O} / \mathbf{I}$ \\
\hline Disgust-Neg & 1.0 & & & & & & & & & & & \\
\hline OBQ & $.48^{*}$ & 1.0 & & & & & & & & & & \\
\hline$R / T$ & $.56^{* * *}$ & $.93^{* *}$ & 1.0 & & & & & & & & & \\
\hline $\mathrm{P} / \mathrm{C}$ & $.43^{*}$ & $.94^{* * *}$ & $.80^{* *}$ & 1.0 & & & & & & & & \\
\hline $\mathrm{I} / \mathrm{CT}$ & .18 & $.67^{* * *}$ & $.52^{* * *}$ & $.49^{* * *}$ & 1.0 & & & & & & & \\
\hline OCI-R & .17 & $.61^{* *}$ & $.63^{* * *}$ & $.71^{* * *}$ & -.004 & 1.0 & & & & & & \\
\hline PI & .34 & $.80^{* *}$ & $.82^{* * *}$ & $.80^{* * k}$ & .23 & $.87^{* *}$ & 1.0 & & & & & \\
\hline CON & .03 & $.52^{* * *}$ & $.50^{* *}$ & $.61^{* *}$ & .03 & $.80^{* * *}$ & $.80^{* * *}$ & 1.0 & & & & \\
\hline$D / G$ & -.01 & $.51^{*}$ & $.46^{*}$ & $.60^{* * *}$ & .12 & $.72^{* *}$ & $.70^{* *}$ & $.80^{* * *}$ & 1.0 & & & \\
\hline CC & $.50^{* * *}$ & $.82^{* * *}$ & $.86^{* * *}$ & $.77^{* * *}$ & $.39 *$ & $.68^{* *}$ & $.83 * *$ & $.39 *$ & .30 & 1.0 & & \\
\hline OT & $.40^{*}$ & $.73^{* * *}$ & $.80^{* * *}$ & $.66^{* *}$ & .31 & $.62^{* *}$ & $.82^{* *}$ & $.45^{*}$ & .36 & $.84^{* * *}$ & 1.0 & \\
\hline OI & .25 & -.04 & .09 & -.09 & -.19 & .03 & .22 & .00 & .009 & .13 & .06 & 1.0 \\
\hline STAI & .16 & $.54^{* * *}$ & $.48^{* * *}$ & $.52^{\#}$ & .36 & .34 & $.47^{* *}$ & .30 & .30 & $.41^{*}$ & $.51^{* * *}$ & .11 \\
\hline
\end{tabular}

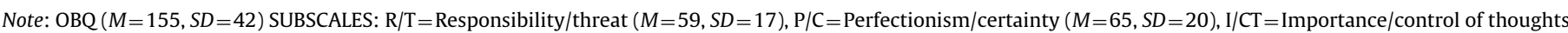
$(M=30, S D=11)$.

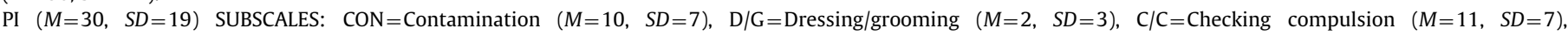
$\mathrm{O} / \mathrm{T}=$ Obsessional thoughts of harm to self/others $(M=5, S D=5), \mathrm{O} / \mathrm{I}=$ Obsessional impulses of harm to self $/$ others $(M=1, S D=3)$. STAI $(M=35, S D=9)$.

$$
\begin{aligned}
& { }^{*} p<.05 . \\
& * * * 01 .
\end{aligned}
$$

Pleasant-Negative Appraisal $(M=.364, S D=.342 ; t(26)=5.53$, $p<.0001)$; Pleasant-Positive Appraisal $(M=.358, S D=.271 ; t(26)=$ $6.88, p<.0001)$.

\subsection{Correlation analysis}

A correlation matrix involving the D-IRAP scores from the Disgust-Negative Appraisal trial-type and all of the explicit measures is presented in Table 2. The majority of the remaining analyses will focus on the Disgust-Negative Appraisal trial-type to assess response biases that reflected negative interpretations to pictures that were chosen to evoke disgust responses. The remaining trial-types will be examined simply to test for divergent validity. Table 2 presents all of the correlations among the explicit measures simply to confirm that the expected large number of inter-correlations was observed. The only notable exception was the absence of any correlation between the Obsessional Impulses of Harm to Self and Others (OI) and any of the other measures. Consistent with the general purpose of the current study, the subsequent set of analyses focused on the correlations among the D-IRAP score and the explicit measures.

The results indicated that there was a medium positive correlation between the D-IRAP scores and the OBQ and two of its subscales, Responsibility/Threat and Perfectionism/Uncertainty. The $D$-IRAP scores failed to correlate with the OCI-R. The relationship between the Padua Inventory and the D-IRAP scores approached significance, and thus its subscales were included in the correlation matrix to determine if any specific factors were driving the correlation toward significance. Two of these five scales, Compulsive Checking and Obsessional Thoughts of Harm to Self/Others, yielded moderate positive correlations with the $D$ IRAP scores. Finally, the state anxiety measure (STAI) failed to correlate with the D-IRAP scores. This pattern of correlations indicates that the more rapidly participants interpret disgusting pictures as being negative, the more highly they scored on two of three general measures of Obsessive-Compulsive tendencies, and on specific sub-scales of these two measures.

A second correlation matrix, which was calculated to assess patterns of responding between the remaining three trial-types and the questionnaires, yielded few interesting results (the full matrix is available for download from the JCBS website). There were two significant negative correlations between the PleasantNegative Appraisal trial-type and the washing concerns subscale of the OCI-R $(r=-.40, p=.03)$ and the contamination subscale of the
Padua Inventory $(r=-.38, p=.05)$. Thus, the greater the implicit response bias towards the pleasant stimuli as not being related to obsessive belief appraisals (i.e., not threatening/their responsibility/perfect etc) the less contamination fear they reported.

\subsection{Predictive validity of the IRAP}

As noted in the Introduction, increased levels of anxiety are often associated with obsessive-compulsive tendencies, and indeed this was confirmed in the pattern of correlations recorded in Table 2 with five significant (and one marginally so) correlations between the anxiety measure and the explicit measures of OCD tendencies. Although the D-IRAP score failed to correlate with anxiety it was deemed important to determine if the implicit measure still predicted obsessive-compulsive tendencies independently of anxiety. Similarly, it was important to determine the predictive validity of the OBQ subscales for scores on the IRAP while controlling for the other subscales to determine which factors are possibly implemented in disgust responding. A series of hierarchical multiple regression analyses were thus conducted to determine the predictive validity of the $D$-IRAP measure while controlling for anxiety and other OC relevant factors (see Table 3). Results indicated that anxiety was a significant predictor of OC tendencies as measured by the OBQ as were the $D$-IRAP scores from the Disgust-Negative Appraisal trial-type when anxiety was controlled for. As can be seen from the subsequent sets of regression analyses (2, 4, 6, and 7), the Disgust-Negative Appraisal trial-type predicted $\mathrm{OC}$ tendencies independent of anxiety in all but one case, Obsessional Thoughts of Harm to Self/Others subscale of the PI. Also, the effect of the DisgustNegative Appraisal trial-type for perfectionism/uncertainty was undermined by responsibility/threat, while perfectionism/uncertainty did not undermine the effect of the IRAP trial-type for responsibility/threat. Similarly, the effect of the perfectionism/ uncertainty factor for negative disgust responding on the IRAP was undermined by the responsibility/threat factor, while perfectionism/uncertainty did not influence the effect of responsibility/threat for disgust responding.

\section{Discussion}

The results of the present study suggest that the IRAP can be used as a measurement of the obsessive beliefs of OCD in 
Table 3

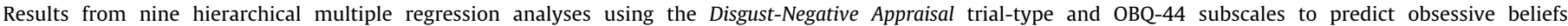
and sub-components of $\mathrm{OC}$ tendencies while controlling for the effects of anxiety and other obsessive beliefs.

\begin{tabular}{|c|c|c|c|}
\hline & B & SE B & Beta \\
\hline \multicolumn{4}{|l|}{ Dependent variable: OBQ } \\
\hline \multicolumn{4}{|l|}{ 1. Step $1\left(R^{2}=.189^{* *}\right)$} \\
\hline Anxiety & 1.90 & .664 & $.435^{* *}$ \\
\hline \multicolumn{4}{|l|}{ Step $2\left(R^{2}\right.$ change $\left.=.144^{* *}\right)$} \\
\hline Disgust-Negative Appraisal Trial-Type & 52.06 & 19.22 & $.380^{* *}$ \\
\hline \multicolumn{4}{|l|}{ Dependent variable: Responsibility/threat $\mathrm{OBQ}$} \\
\hline \multicolumn{4}{|l|}{ 2. Step $1\left(R^{2}=.178^{* * *}\right)$} \\
\hline Anxiety & .777 & .283 & $.421^{* *}$ \\
\hline \multicolumn{4}{|l|}{ Step $2\left(R^{2}\right.$ change $\left.=.225^{* *}\right)$} \\
\hline Disgust-Negative Appraisal Trial-Type & 27.52 & 7.69 & $.475^{* *}$ \\
\hline \multicolumn{4}{|l|}{ Step $1\left(R^{2}=.508^{* * * * *}\right)$} \\
\hline Perfectionism/certainty & .636 & .106 & $.713^{* * * *}$ \\
\hline \multicolumn{4}{|l|}{ Step $2\left(R^{2}\right.$ change $\left.=.059^{*}\right)$} \\
\hline Disgust-Negative Appraisal Trial-Type & 15.13 & 7.02 & $.261^{*}$ \\
\hline \multicolumn{4}{|c|}{ Dependent variable: Perfectionism/certainty OBQ } \\
\hline \multicolumn{4}{|c|}{ 4. Step $1\left(R^{2}=.084\right)$} \\
\hline Anxiety & .599 & .334 & .290 \\
\hline Step $2\left(R^{2}\right.$ change $\left.=.127^{*}\right)$ & & & \\
\hline Disgust-Negative Appraisal Trial-Type & 23.16 & 9.90 & $.357 *$ \\
\hline \multicolumn{4}{|l|}{ Step $1\left(R^{2}=.508^{* * * *}\right)$} \\
\hline Responsibility/Threat & .799 & .133 & $.713^{* * * *}$ \\
\hline \multicolumn{4}{|l|}{ Step $2\left(R^{2}\right.$ change $\left.=.000\right)$} \\
\hline Disgust-Negative Appraisal Trial-Type & 1.52 & 8.94 & .023 \\
\hline \multicolumn{4}{|l|}{ Dependent variable: Checking PI } \\
\hline \multicolumn{4}{|l|}{ 6. Step $1\left(R^{2}=.186^{* * *}\right)$} \\
\hline Anxiety & .333 & .118 & $.431^{* *}$ \\
\hline \multicolumn{4}{|l|}{ Step $2\left(R^{2}\right.$ change $\left.=.168^{* *}\right)$} \\
\hline Disgust-Negative Appraisal Trial-Type & 9.99 & 3.36 & $.410^{* * *}$ \\
\hline \multicolumn{4}{|c|}{ Dependent variable: Obsessional thoughts of harm to self/others subscale of PI } \\
\hline \multicolumn{4}{|c|}{ 7. Step $1\left(R^{2}=.311^{* * * *}\right)$} \\
\hline Anxiety & .318 & .080 & $.55^{* * * *}$ \\
\hline \multicolumn{4}{|l|}{ Step $2\left(R^{2}\right.$ change $\left.=.039\right)$} \\
\hline Disgust-Negative Appraisal Trial-Type & 3.55 & 2.48 & .198 \\
\hline \multicolumn{4}{|c|}{ Dependent variable: Disgust-Negative Appraisal Trial-Type } \\
\hline \multicolumn{4}{|c|}{ 8. Step $1\left(R^{2}=.236^{* * *}\right)$} \\
\hline Responsibility/threat & .008 & .003 & $.486^{* *}$ \\
\hline Step $2\left(R^{2}\right.$ change $\left.=.001\right)$ & & & \\
\hline Perfectionism/certainty & .001 & .003 & .036 \\
\hline Step $1\left(R^{2}=.133^{*}\right)$ & & & \\
\hline Perfectionism/certainty & .006 & .002 & $.364 *$ \\
\hline Step $2\left(R^{2}\right.$ change $\left.=.104^{*}\right)$ & & & \\
\hline Responsibility/Threat & .008 & .004 & $.460^{*}$ \\
\hline
\end{tabular}

$* p \leq .05$
$* * *<<.01$

$* * * * k<.000$.

response to disgust-eliciting stimuli. A greater bias toward interpreting the disgusting stimuli as being negative predicted overall scores on the OBQ and specifically scores on the responsibility/ threat and perfectionism/uncertainty subscales of the OBQ. Additionally, scores on the checking subscale of the Padua Inventory were predicted by the IRAP. Critically, these effects (from both the OBQ and the Padua Inventory) were independent of current levels of anxiety. One effect which was influenced by anxiety was the relationship between scores on the IRAP and the obsessive thoughts of harm to the self/others subscale of the PI. The impact of OC relevant beliefs such as responsibility/threat and perfectionism/uncertainty on the predictive validity of the IRAP were also examined. The regression analyses indicated that the DisgustNegative Appraisal trial-type from the IRAP failed to predict perfectionism/uncertainty when controlling for responsibility/ threat. However, when controlling for perfectionism/uncertainty, the IRAP still predicted responsibility/threat, which further highlights the role of the latter factor in disgust responding. The lack of any meaningful relationships between the other trial-types and self-report measures was indicative of the discriminant validity of the IRAP in that only the OC relevant trial-type (Disgust-Negative Appraisal) was related to obsessive beliefs and other obsessivecompulsive constructs.

The IRAP used in the current study was not specifically designed to measure checking compulsions; however, the Disgust-Negative Appraisal trial-type predicted scores on the checking subscale of the PI, independently of anxiety. This finding suggests that the IRAP was tapping into OC-relevant cognitions beyond those measured in the $\mathrm{OBQ}$ on which it was based. A possible explanation of this finding could be that checking is often viewed as a by-product of perfectionism and as a precursor for uncertainty which is intolerable to OCD sufferers. Repetitive checking behaviors, which are characteristic to OCD sufferers, have been demonstrated to facilitate greater uncertainty due to a reduction in the vividness and trustworthiness of memory (van den Hout \& Kindt, 2004). Similarly, perfectionism, which is often motivated by a desire to avoid highly unlikely catastrophic events, could be manifested in the form of compulsive checking behaviors to prevent a negative event from occurring (Bouchard, Rheaume \& Ladouceur, 1999). Indeed, perfectionism has been shown to play a specific role in the maintenance of checking 
compulsions and this effect was mediated by anxiety (Moretz \& McKay, 2009). It could be argued that, based on the relationship of the Disgust-Negative Appraisal trial-type to checking, that the present IRAP was indeed measuring additional cognitions and biases that are relevant to OCD.

Previous research has found that responsibility appraisals mediated the role of perfectionism on checking and cleaning symptoms in a normal sample (Yorulmaz, Karancı \& Tekok-Kıll, 2006). These results somewhat reflect the finding in the present study that the responsibility/threat factor negatively influenced the effect of the perfectionism/uncertainty factor for disgust responding as measured by the IRAP. The results obtained by Yorulmaz et al. (2006) drew distinctions between self and socially orientated perfectionism (see Hewitt \& Flett, 1991); that is responsibility mediated the effect of self-orientated perfectionism for checking and socially-orientated perfectionism for cleaning. Based on the statements in the OBQ the perfectionism subscale appears to be measuring both self and socially orientated perfectionism. A possible explanation for the similarity in findings between the present study and Yorulmaz et al. (2006) is that cleaning behaviors, along with checking, could be motivated by a heightened sense of disgust sensitivity which could be why responsibility/threat influenced the effect of perfectionism/ uncertainty for disgust.

An inflated sense of personal responsibility has long been considered a vital component of OCD (Salkovskis, 1985, 1989) and the present results appear to add to existing evidence which suggests that it may be critical in problematic disgust responding also. Indeed, previous research has found that the responsibility/ threat subscale was predictive of contamination symptoms in an OCD population (Wheaton, Abramowitz, Berman, Riemann \& Hale, 2010). Teachman (2006) posited that maladaptive disgust responding could be interpreted in terms of excessive responsibility for contamination prevention. That is, an individual who has a greater sensitivity to disgust could interpret disgusting or contaminated objects in the environment as being their responsibility to clean up or eliminate (e.g., "When I see any opportunity to do so, I must act to prevent bad things from happening"). However, insofar as the responsibility/threat factor in the $\mathrm{OBQ}$ measures both constructs in one subscale, further research examining this relationship more thoroughly is needed to determine the extent to which responsibility and threat individually influence disgust responding.

Previous research using the most popular measure of implicit attitudes, the Implicit Association Test (IAT; Greenwald, McGhee \& Schwartz, 1998), failed to find a significant relationship between the implicit appraisal of unwanted thoughts as being personally meaningful and the cognitive belief domains as measured by the OBQ (Teachman, Woody \& Magee, 2006). However, it is worth noting that the IAT in this study used worded stimuli such as "Unwanted Thoughts" and "Wanted Thoughts" and a list of words to categorize each type of thought such as "Important" or "Meaningful" (Teachman et al., 2006). That is, there was no reference to the specific content of the unwanted thoughts. Rachman \& De Silva (1978) have demonstrated that the majority of people (both OCD and non-OCD) experience similar intrusive thoughts in terms of content. The present results using the IRAP, which presented specific disgust-eliciting stimuli, suggest that the content of intrusive and unwanted thoughts may be critical when measuring cognitive belief domains at the implicit level.

Additionally, a detailed examination of the items in the OBQ brings to light the propositional nature of the measure (e.g., "If I don't act when I foresee danger, then I am to blame for any consequences"). The IAT was designed as a measure of the strength of associations between concepts in memory and it is widely accepted that IAT effects do not reflect propositional processes (see De Houwer, 2002, for a detailed explanation). In contrast, the IRAP is based on a theoretical framework of cognition that assumes that implicit cognition is highly relational or propositional in nature (Hughes et al., 2011), and indeed the IRAP was specifically designed to measure the relational properties of implicit cognition (e.g. Nicholson \& Barnes-Holmes, 2012a). Thus, the finding that scores on the IRAP were related to scores on the OBQ while the IAT were not supports the use of the IRAP as a measure of conditional beliefs such as those observed in OCD.

One possible limitation of the current study is that participants may have been responding to the pictorial stimuli as merely positive or negative rather than disgusting or non-disgusting. Nevertheless, this is unlikely given the verbal context that was provided during instruction. Future research could attempt to utilize negative stimuli, both disgusting and non-disgusting, however due to the multidimensional nature of disgust, it would be difficult to acquire negative pictures that could in no way be interpreted as disgusting in some sense. For instance, items that represent immoral behavior (i.e., a picture of a swastika or pictures of criminals) have been known to induce moral disgust (Jones \& Fitness, 2008).

While evidence suggests that $O C$-related responding can be recorded in a non-clinical population (Burns et al., 1995), a general limitation of the present study was the use of a nonclinical student sample. The means and standard deviations of the self-report measures of OCD and their subscales for the present sample as a whole were slightly higher than those obtained by previous research for a student sample, however, were within one standard deviation (e.g., Burns et al., 1995; Obsessive Compulsive Cognitions Working Group, 2005). Moreover, thirteen of the participants produced scores comparable to those produced by a clinical sample of individuals with a diagnosis of OCD (Obsessive Compulsive Cognitions Working Group, 2005). This is not unusual given that student samples have previously been found to produce higher scores on OCD measures such as the OBQ than average community samples, which further supports their use in such research (Obsessive Compulsive Cognitions Working Group, 2005). Nevertheless, it would be expected that a clinical sample would produce higher scores than a student sample. Further research comparing a clinical sample of patients with a diagnosis of OCD with a non-patient control sample would provide further insight into the relationship between disgust and the obsessive belief domains of OCD.

\section{Conclusions}

The current study provides further evidence to add to the growing literature delineating the relationship between disgust and OCD. Specifically, the present results parse out the individual effect of disgust independently of anxiety, which is vital if disgust is to be viewed as a significant emotion in its own right. The results further explicate the role of each belief domain in disgust responding providing greater insight into the cognitive mechanisms through which disgust can influence OCD. Teachman (2006) emphasized the importance of secondary appraisals or interpretations for clarifying findings in the literature regarding disgust and the anxiety disorders. While more research is needed, the evidence in the literature is becoming more suggestive that the negative interpretation of the initial intrusive feeling of disgust can have extremely damaging consequences, particularly in OCD. Wheaton et al. (2010) called for behavioral measures of OCD in order to circumvent the pitfalls of working exclusively with selfreport measures and the IRAP appears to offer a novel way of measuring these cognitive beliefs relevant to OCD. While the use of the IRAP in this domain is in the early stages, and further research assessing its use at different symptomatic levels is needed, the results from the current research appear promising. 


\section{Role of funding sources}

Funding for this study was provided by the John and Pat Hume Scholarship Scheme and the Irish Research Council awarded to the first author.

\section{References}

Barnes-Holmes, D., Barnes-Holmes, Y., Stewart, I., \& Boles, S. (2010). A sketch of the Implicit Relational Assessment Procedure (IRAP) and the relational elaboration and coherence (REC) model. Psychological Record, 60, 527-542.

Barnes-Holmes, D., Hayes, S. C., Dymond, S., \& O’Hora, D. (2001). Multiple stimulus relations and the transformation of stimulus functions. In: S. C. Hayes, D. Barnes-Holmes, \& B. Roche (Eds.), Relational Frame Theory: A post- Skinnerian account of human language and cognition. New York: Kluwer/Plenum.

Bouchard, C., Rheaume, J., \& Ladouceur, R. (1999). Responsibility and perfectionism in OCD: An experimental study. Behaviour Research and Therapy, 37, 239-248.

Burns, G. L., Formea, C. L., Koertege, S., \& Sternberger, L. G. (1995). The utilisation of non-patient samples in the study of obsessive compulsive disorder. Behaviour Research and Therapy, 33, 133-144.

Burns, G. L., Koertege, S., Formea, C. L., \& Sternberger, L. G. (1996). Revisions of the Padua Inventory of obsessive-compulsive disorder symptoms: Distinctions between worry, obsessions, and compulsions. Behaviour Research and Therapy, 34, 163-173.

Carpenter, K. M., Martinez, D., Vadhan, N. P., Barnes-Holmes, D., \& Nunes, E. V. (2012). Measures of attentional bias and relational responding are associated with behavioral treatment outcome for cocaine dependence. American Journal of Drug and Alcohol Abuse, 38, 146-154.

Cisler, J. M., Brady, R. E., Olatunji, B. O., \& Lohr, J. M. (2010). Disgust and obsessive beliefs in contamination-related OCD. Cognitive Therapy and Research, 34, 439-448.

David, B., Olatunji, B. O., Armstrong, T., Ciesielski, B. C., Bondy, C. L., \& BromanFulks, J. (2009). Incremental specificity of disgust sensitivity in the prediction of obsessive-compulsive disorder symptoms: Cross-sectional and prospective approaches. Journal of Behavior Therapy and Experimental Psychiatry, 40, 533-543.

Dawson, D. L., Barnes-Holmes, D., Gresswell, D. M., Hart, A J. P., \& Gore, N. J. (2009). Assessing the implicit beliefs of sexual offenders using the implicit relational assessment procedure: A first study. Sexual Abuse: A Journal of Research and Treatment, 21, 57-75.

De Houwer, J. (2002). The Implicit Association Test as a tool for studying dysfunctional associations in psychopathology: Strengths and limitations. Journal of Behavior Therapy and Experimental Psychiatry, 33, 115-133.

Foa, E. B., Huppert, J. D., Leiberg, S., Langner, R., Kichic, R., Hajcak, G., et al. (2002). The obsessive-compulsive inventory: Development and validation of a short version. Psychological Assessment, 14 485-469.

Greenwald, A. G., McGhee, D. E., \& Schwartz, J. L. K. (1998). Measuring individual differences in implicit cognition: The implicit association test. Journal of Personality and Social Psychology, 78, 1464-1480.

Greenwald, A. G., Nosek, B. A., \& Banaji, M. R. (2003). Understanding and using the implicit association test: I. An improved scoring algorithm. Journal of Personality and Social Psychology, 85, 197-216.

Haidt, J., McCauley, C., \& Rozin, P. (1994). Individual differences in sensitivity to disgust: A scale sampling seven domains of disgust elicitors. Personality and Individual Differences, 16, 701-713.

Hayes, S. C., Barnes-Holmes, D., \& Roche, B. (2001). Relational frame theory: A postskinnerian account of human language and cognition. New York: Kluwer/ Plenum.

Hewitt, P. L. \& Flett, G. L. (1991). Perfectionism in the self and social contexts: Conceptualization, assessment, and association with psychopathology. Journal of Personality and Social Psychology, 60, 456-470.

van den Hout, M., \& Kindt, M. (2004). Obsessive-compulsive disorder and the paradoxical effects of perseverative behavior on experienced uncertainty. Journal of Behavior Therapy and Experimental Psychiatry, 35, 165-181.

Hughes, S., Barnes-Holmes, D., \& De Houwer, J. (2011). The dominance of associative theorising in implicit attitude research: Propositional and behavioral alternatives. Psychological Record, 61, 465-496.

Jones, A., \& Fitness, J. (2008). Moral hypervigilance: The influence of disgust sensitivity in the moral domain. Personality and Individual Differences, 8, 613-627.

Lang, P. J., Bradley, M. M., \& Cuthbert, B. N. (1996). International affective picture system (IAPS): Technical manual and affective ratings. NIMH Center for the Study of Emotion and Attention.

McKenna, I. M., Barnes-Holmes, D., Barnes-Holmes, Y., \& Stewart, I. (2007). Testing the fakeability of the Implicit Relational Assessment Procedure (IRAP): The first study. International Journal of Psychology and Psychological Therapy, 7, $123-138$

Moretz, M. W., \& McKay, D. (2008). Disgust sensitivity as a predictor of obsessive compulsive contamination symptoms and associated cognitions. Journal of Anxiety Disorders, 22, 707-715.

Moretz, M. W., \& McKay, D. (2009). The role of perfectionism in obsessivecompulsive symptoms: "Not just right" experiences and checking compulsions. Journal of Anxiety Disorders, 23, 640-644.

Nicholson, E., \& Barnes-Holmes, D. (2012a). Developing an implicit measure of disgust propensity and disgust sensitivity: Examining the role of implicit disgust propensity and sensitivity in obsessive compulsive tendencies. Journal of Behavior Therapy and Experimental Psychiatry, 43, 922-930.

Nicholson, E., \& Barnes-Holmes, D. (2012b). The Implicit Relational Assessment Procedure (IRAP) as a measure of spider fear. Psychological Record, 62, 263-278.

Nosek, B., Greenwald, A. G., \& Banaji, M. R. (2007). The implicit association test at age 7: A methodological and conceptual review. In: J. A. Bargh (Ed.), Automatic processes in social thinking and behavior. Psychology Press.

Obsessive Compulsive Cognitions Working Group (1997). Cognitive assessment of obsessive compulsive disorder. Behaviour Research and Therapy, 35, 667-681.

Obsessive Compulsive Cognitions Working Group (2001). Development and initial validation of the obsessive beliefs questionnaire and the interpretation of intrusions inventory. Behaviour Research and Therapy, 39, 987-1006.

Obsessive Compulsive Cognitions Working Group (2005). Psychometric validation of the obsessive beliefs questionnaire and interpretation of intrusions inventory: Part 2, factor analyses and testing of a brief version. Behaviour Research and Therapy, 43, 1527-1542.

van Overveld, W. J. M., De Jong, P. J., Peters, M. L., Cavanagh, K., \& Davey, G. C. L (2006). Disgust propensity and disgust sensitivity: Separate constructs that are differentially related to specific fears. Personality and Individual Differences, $41,1241-1252$

van Overveld, W. J. M., De Jong, P. J., Peters, M. L., \& Schouten, E. (2011). The disgust scale-R: A valid and reliable index to investigate separate disgust domains? Personality and Individual Differences, 51, 325-330.

Olatunji, B. O., Williams, N. L., Lohr, J. M., Connelly, K. M., Cisler, J., \& Meunier, S. A (2007). Structural differentiation of disgust from trait anxiety in the prediction of specific anxiety disorder symptoms. Behaviour Research and Therapy, 45, 3002-3017.

Rachman, S. (1997). A cognitive theory of obsessions. Behaviour Research and Therapy, 35, 793-802

Rachman, S. (1998). A cognitive theory of obsessions: Elaborations. Behaviour Research and Therapy, 36, 385-401.

Rachman, S., \& De Silva, P. (1978). Abnormal and normal obsessions. Behaviour Research and Therapy, 16, 233-248.

Salkovskis, P. (1985). Obsessional compulsive problems: A cognitive behavioura analysis. Behaviour Research and Therapy, 23, 571-583.

Salkovskis, P. (1989). Cognitive-behavioural factors and the persistence of intrusive thoughts in obsessional problems. Behaviour Research and Therapy, 27, 677-682.

Salkovskis, P., Shafran, R., Rachman, S., \& Freeston, M. H. (1999). Multiple pathways to inflated responsibility beliefs in obsessional problems: Possible origins and implications for therapy and research. Behaviour Research and Therapy, 37, 1055-1072.

Spielberger, C. D., Gorsuch, R. L., Lushene, R., Vagg, P. R., \& Jacobs, G. A. (1983) Manual for the state-trait anxiety inventory. Palo Alto, CA: Consulting Psychologists Press.

Teachman, B. A. (2006). Pathological disgust: In the thoughts, not the eye, of the beholder. Anxiety, Stress, and Coping, 19, 335-351.

Teachman, B. A., Woody, S. R., \& Magee, J. C. (2006). Implicit and explicit appraisals of the importance of intrusive thoughts. Behaviour Research and Therapy, 44 785-805.

Twohig, M. P. (2009). The application of acceptance and commitment therapy to obsessive-compulsive disorder. Cognitive and Behavioral Practice, 16, 18-28.

Vahey, N. A., Barnes-Holmes, D., Barnes-Holmes, Y., \& Stewart, I. (2009). A first test of the Implicit Relational Assessment Procedure as a measure of self-esteem: Irish prisoner groups and university students. The Psychological Record, 59, 371-388.

Wheaton, M. G., Abramowitz, J. S., Berman, N. C., Riemann, B. C., \& Hale, L. R. (2010). The relationship between obsessive beliefs and symptom dimensions in obsessive-compulsive disorder. Behaviour Research and Therapy, 48 949-954.

Wiers, R. W., Teachman, B. A., \& De Houwer, J. (2007). Implicit cognitive processes in psychopathology: An introduction. Journal of Behavior Therapy and Experimental Psychiatry, 38, 95-104.

Wu, K. D., \& Carter, S. A. (2008). Further investigation of the obsessive beliefs questionnaire: Factor structure and specificity of relations with OCD symptom. Journal of Anxiety Disorders, 22, 824-836.

Yorulmaz, O., Karancı, A. N., \& Tekok-Kılı, A. (2006). What are the roles of perfectionism and responsibility in checking and cleaning compulsions? Journal of Anxiety Disorders, 20, 312-327. 